\title{
Michael Franz Hat die Lebensqualitätsforschung ihren Zweck erfüllt?
}

\author{
Did Quality of Life Research Achieve its Aim?
}

"Quality of Life“ nahm bis in die späten 90er-Jahre einen festen Platz innerhalb der großen Kongressthemen ein. Seit der Jahrtausendwende verschwindet ihre explizite wissenschaftliche Thematisierung, ihre Anwendung als Indikator zur Bewertung psychiatrischer Therapie ist breit etabliert und nimmt kontinuierlich zu (Abb.1). Zugleich existiert noch keine einheitliche Methode, Lebensqualität zu bestimmen [1], bestehen ungelöste konzeptuelle Probleme und „kontraintuitive“ Lebensqualitätsbefunde, die den Betroffenen potenziell mehr schaden können als nützen [1 -4]. Daher wird in Anlehnung an einen Satz von Stefan Priebe [5] gefragt: Haben sich die Erwartungen an die Einführung eines subjektiven Indikators erfüllt? Wem nutzt die Messung von Lebensqualität heute?

\section{Sozialpsychiatrie und das Lebensqualitätskonzept - zur Notwendigkeit einer Enttäuschung}

Im Kontext der Erkenntnis, dass die chronisch psychisch Kranken trotz Reform der objektiven „Anstalts“-Lebensbedingungen nun in der Gemeinde chronifizierten [6], findet sich eine Zunahme der Arbeiten zur subjektiven Perspektive der Patienten und Angehörigen [7]. Diese hatte zuvor in der quantitativen psychiatrischen Evaluationsforschung eine untergeordnete Rolle gehabt. Der ausschlaggebende Faktor für die Rezeption des Lebensqualitätskonzepts in der Sozialpsychiatrie ist jedoch, dass es keine neue Perspektive darstellte. Die subjektive Perspektive hat im Gegensatz zur Organmedizin in der Sozialpsychiatrie traditionell allerhöchsten Stellenwert, nicht nur wegen der psychiatrischen Alltagspraxis [1]. Subjektorientierte Ansätze wie Phänomenolo- gie [8] und Daseinsanalyse bestimmten lange die psychiatrische Erkenntnistheorie [9]. Vertreter der Psychiatriereform grenzten sich schon früh von einer als naturwissenschaftlich - objektivierend empfundenen Schul- oder Anstalts-Psychiatrie ab, indem sie das „Subjektsein“ der Patienten in den Vordergrund rückten [10]. Diese Perspektive hat den Reformgruppierungen seit den 70er-Jahren Identität und eine kämpferische Perspektive ermöglicht. Daher hat der Begriff „Lebensqualität“ für die Sozialpsychiatrie keine neue Perspektive eröffnet, sondern ein vorhandenes Grundbedürfnis kristallisiert. „Lebensqualität‘ fasst zusammen, was man mit einem vagen Vorverständnis schon immer meinte“ [11].

Neu war, die subjektive Perspektive systematisch, umfassend und gleichzeitig zeitökonomisch als evaluativen Index in wissenschaftliche Fragestellungen einbinden zu wollen. Dabei bestand die Erwartung, einen Indikator zu gewinnen, der (benachteiligende) objektive Bedingungen so abbildet, dass die durch ihn gewonnenen subjektiven Informationen für die Planung und Bewertung von Versorgungsangeboten objektiven Daten überlegen sind oder sie entscheidend bereichern. In diesem Kontext bekam die Anwendung als Outcome-Indikator den Charakter eines politisch korrekten, quasi-emanzipatorischen Aktes, um das Dasein bzw. die Versorgungslage einer benachteiligten Gruppe zu verbessern. Paradigmatisch formulierten dies Baker u. Intagliata [12]:

1. Da psychiatrische Krankheiten unheilbar seien, sei das Leben mit der Krankheit so angenehm wie möglich zu gestalten. Besonders bei psychiatrischen Langzeitpatienten sei unrealistisch, eine enorme Verbesserung der Symptomatik anzustre- 


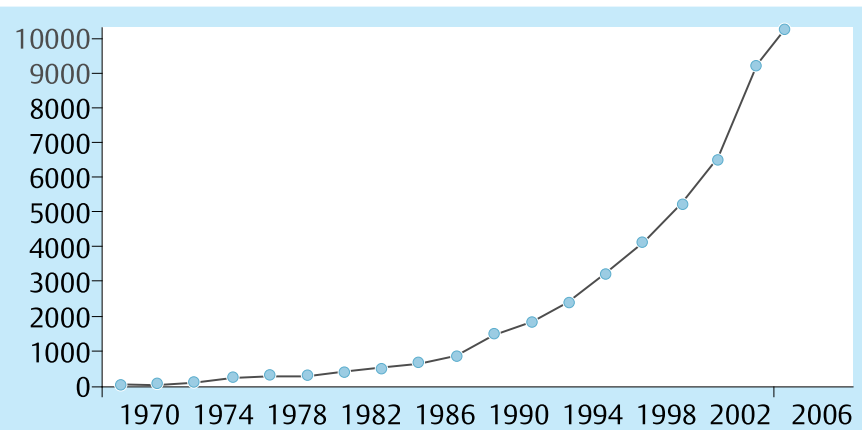

Abb. 1 Medline: Suchbegriff Lebensqualität und Schizophrenie/und Depression/und Psychiatrie im Abstract. Kumulierte Häufigkeit von Publikationen.

ben. Vielmehr solle den Patienten ein möglichst angenehmes Leben ermöglicht werden.

2. Da es sich bei Lebensqualität um ein „multidimensionales Konstrukt" handele, sei es möglich, komplexe Interventionsprogramme zu evaluieren.

3. Die Interessen der Betroffenen träten mehr in den Mittelpunkt der Beurteilung verschiedener Interventionen, es komme zu einer höheren „Kundenorientierung“.

4. Das Konzept der Lebensqualität ermögliche, die gesamten Lebensbedingungen einer Person zu erheben, statt einzelne Punkte, wie beispielsweise die Psychopathologie, zu fokussieren.

5. Der Begriff der Lebensqualität werde in politischen Diskussionen aufgegriffen.

Die Anwendung von subjektiver Lebensqualität als Indikator steht hier im Kontext eines Gesamtkonzeptes zur Verbesserung des Daseins (i.S.v. well-being“1 ${ }^{\prime \prime}$ psychisch Kranker (Punkt 2. und 4.). Abgesehen davon, dass sich unter Punkt 4 Abgrenzungsprobleme gegenüber bekannten und in sich besser abgegrenzten Konstrukten ergeben [1], sind die beiden erfassungsökonomischen Anforderungen konfliktträchtig: der Indikator soll als evaluativer Index auf einen „Punkt“ bzw. Zahl gebracht werden (Punkt 2) und gleichzeitig allumfassend sein (Punkt 4).

In der Folge etablierte sich keine breite wissenschaftliche Auseinandersetzung, sondern eine routinemäßige Lebensqualitätserfassung in einer unüberschaubaren Menge von Anwendungen $[1,3]$. In der Praxis wurden Lebensqualitätsinstrumente ohne die einfachsten konzeptuellen Präzisierungen eingesetzt. Man ließ nach dem Bekanntheitsgrad ausgewählte Skalen „mitlaufen“, um die „subjektive Seite“ irgendwie zu berücksichtigen. Schwer interpretierbare Ergebnisse [3], die den Betroffenen durchaus schaden konnten [3,4], stehen im Widerspruch zum ursprünglichen reformerischen Interesse. Diese - sicher ungewollte - Entwicklung ist durch mehrere, konvergierende Faktoren zu erklären:

1. Die o.g. idealisierende Erwartung, dass bereits in einer Anwendung von Lebensqualitätsskalen ein reformerisches Potenzial liegt.

\footnotetext{
${ }^{1}$ Well-being wird als Wohlbefinden übersetzt, bezeichnet im Englischen aber ebenso Wohlfahrt (gutes „Sein“, Zustand „guter“ Wohlfahrt, vgl. [16]).
}

2. Fragebogenstudien sind relativ leicht durchführbar und die Bedingungen einer akademischen Umgebung durch ein „publish or perish“-Prinzip dominiert.

3. Mittelwerte von Lebensqualitätsskalen werden in guten wissenschaftlichen Journalen ohne weitere theoretische Explikation als Maß für „die“ selbstwahrgenommene Lebensqualität schizophrener Patienten bezeichnet, transkulturelle Adaptationen in immer weiteren Ländern publiziert, um die Effizienz ihrer Versorgungssysteme zu beurteilen. „Diagnostische Verfahren zu Lebensqualität“" [13] werden als Sammlung herausgegeben und die Verwendung von Lebensqualitätsskalen ist Auflage zur Zulassung von Neuroleptika. Dieses Szenario kann auf Anwender den Eindruck entstehen lassen, „dass es sich um ein hinreichend etabliertes Konstrukt handelt, welches an sich nicht mehr grundlegend erforscht werden muss, sondern für Fragestellungen außerhalb seiner selbst funktionalisiert werden kann“ [1].

\section{Wem nutzt die Erfassung von Lebensqualität?}

Den psychisch Kranken selbst ist die beschriebene Entwicklung zunächst zugute gekommen. Ihre subjektive Sichtweise bekam - auch außerhalb der Sozialpsychiatrie - einen Stellenwert, den sie nie zuvor hatte. Sie wurden von passiven Behandlungsobjekten zu aktiven Partnern, treffen individuelle Behandlungsvereinbarungen mit Krankenhäusern, besitzen organisiert mehr politischen Einfluss als früher und werden an der Konzeption von Versorgung und der Vergabe von Forschungsgeldern beteiligt [14].

Der Forschung hat die stärkere Berücksichtigung der subjektiven Perspektive unter dem Dach der Lebensqualitätsforschung zu einer außergewöhnlichen wissenschaftlichen Auseinandersetzung mit der Patientenperspektive verholfen, die viele offene Forschungsfragen stimuliert [15].

Somit hat die Lebensqualität auf vielen Ebenen ihren Zweck erfüllt, auf denen das Erreichte weiter vorangetrieben werden muss. Das Ziel einer substanziellen Erweiterung der Indikatoren psychiatrischer Therapien ist nicht erreicht (vgl. die Beiträge von Pukrop, Kilian und Franz in diesem Heft).

\section{Fazit}

Nicht Lebensqualität ist problematisch, sondern ihre kritiklose Anwendung. Es ist an der Zeit, letztere zu beenden und aus ihren Fehlern zu lernen. Forschungsaktivitäten sind wieder auf Theoriebildung, Grenzen und Möglichkeiten des Konzepts selbst sowie einzelner, unter dem Dach des Begriffes Lebensqualität zur Anwendung kommender Konstrukte zu konzentrieren, statt immer weiter Lebensqualitätsskalen „mitlaufen“ zu lassen.

Mentale Verknüpfungen zwischen reformerisch-emanzipatorischem Impetus und dem Messen subjektiver Lebensqualität müssen gelöst, die Hoffnung, subjektive Lebensqualität könne als „besserer“ oder "humanerer“ Indikator allein durch ihre Anwendung zu einer optimaleren Evaluation oder valideren Abbildung der Bedürfnisse psychisch Kranker führen, aufgegeben werden. Sozialpsychiatrie soll politisch und in der Konzeption 
von Versorgung für die Patienten kämpfen. Aber es ist kein primär politisch korrekter, emanzipatorischer Akt, sondern eine besondere methodische und theoretische Herausforderung, Lebensqualität als Indikator anzuwenden. Wir sollten uns ihr stellen.

\section{Literatur}

${ }^{1}$ Pukrop R. Subjektive Lebensqualität. Kritische Betrachtung eines modernen Konstruktes. Nervenarzt 2003; 74: 48-54

2 Roick C, Angermeyer MC. Ökonomie und Praxis. Psychiat Prax 2004; 31: $115-117$

${ }^{3}$ Kilian R. Ist Lebensqualität messbar? Probleme der quantitativen und Möglichkeiten der qualitativen Erfassung von Lebensqualität in der Psychiatrie. Psychiat Prax 1995; 22: 97 - 101

${ }^{4}$ Franz M, Meyer T, Gallhofer B. Subjektive Lebensqualität schwer chronifizierter schizophrener Langzeitpatienten. Teil 3 der Hessischen Enthospitalisierungsstudie. Psychiat Prax 2002; 29: 306-310

${ }^{5}$ Priebe S. Haben Enthospitalisierungsstudien ihren Zweck erfüllt? Psychiat Prax 2001; 28: 207-208

${ }^{6}$ Hoffmann $\mathrm{H}$. Junge chronische Patienten: Wie können wir die schwierigsten unter ihnen besser behandeln? Nervenarzt 1993; 64: 62 - 69
${ }^{7}$ Holzinger A, Angermeyer MC. Sozialpsychiatrische Forschung im deutschen Sprachraum. Psychiat Prax 2002; 29: 397-410

${ }^{8}$ Jaspers K. Allgemeine Psychopathologie (4. Auflage). Heidelberg: Springer, 1946

${ }^{9}$ Schmidt-Degenhard M, Feldmann H. Hermeneutische Psychopathologie der Psychosen: Wissenschaftstheoretische Grundlegung - Konzepte - Klinische Aspekte. Nervenarzt 2003; 74: 16-22

${ }^{10}$ Keupp H. Das Subjekt der Postmoderne - zwischen Multiphrenie und Selbstsorge. Psychiat Prax 2003; 30 (Suppl 1): S3-S13

${ }^{11}$ Pöppel E. Lebensqualität: Zur Herausforderung eines Begriffes. In: Bullinger M, Ludwig M, Steinbüchel N von (Hrsg): Lebensqualität bei kardiovaskulären Erkrankungen. Göttingen: Hogrefe, 1991: 1-3

12 Baker F, Intagliata J. Quality of life in the evaluation of community support systems. Eval Program Plann 1982; 5: 69-79

${ }^{13}$ Schumacher J, Klaiberg A, Brähler E(Hrsg). Diagnostische Verfahren zu Lebensqualität und Wohlbefinden. Göttingen: Hogrefe, 2003: 1-16

${ }^{14}$ Priebe S. Zukunft psychiatrischer Versorgung. Träume und Alpträume. Psychiat Prax 2003; 30 (Suppl 1): S48-S53

${ }^{15}$ Fischer M, Kemmler G, Meise U. Burden - Distress - Lebensqualität. Psychiat Prax 2004; 31: 57-59

${ }^{16}$ Meyer T. Beiträge eines strukturierten, offenen Interviews zur Erfassung des Konstrukts Subjektive Lebensqualität bei schizophrenen Menschen. Hamburg: Verlag Dr. Kovac, 2004: 12 